\title{
Early Onset Benign Occipital Epilepsy (Panayiotopoulos syndrome): a case Report \& Literature Review
}

\author{
Md Enayet Hussain¹, Rajib Nayan Chowdhury², Md Nahidul Islam³, AFM Al \\ Masum Khan ${ }^{4}$, Md. Ferdous Miah ${ }^{5}$ \\ ${ }^{1}$ Assistant Professor, Department of Neurology, National Institute of Neurosciences \& Hospital, Dhaka, Bangladesh; \\ ${ }^{2}$ Assistant Professor, Department of Neurophysiolology, National Institute of Neurosciences \& Hospital, Dhaka, \\ Bangladesh; ${ }^{3}$ Registrar, Department of Neurophysiology, National Institute of Neurosciences \& Hospital, \\ Dhaka, Bangladesh; ${ }^{4}$ Medical Officer, Department of Neurophysiology, National Institute \\ of Neurosciences \& Hospital, Dhaka, Bangladesh; ${ }^{5}$ Medical Officer, Department of \\ Neurophysiology, National Institute of Neurosciences \& \\ Hospital, Dhaka, Bangladesh
}

Received on: 21 July 2014; Reviewed on: 6 September 2014; Published on: 1 January 2015

\begin{abstract}
Panayiotopoulos syndrome is a type of benign childhood epilepsy syndrome characterized by episodic autonomic and mainly emetic symptoms. It predominantly affects children of 3-6 years of age (13\% of those with one or more non-febrile seizures). EEG shows great variability, with occipital, extra-occipital spikes or brief generalised discharges alone or in combination; it may also be consistently normal. Despite the high prevalence of autonomic status epilepticus, the prognosis of Panayiotopoulos syndrome is usually excellent and most do not require anti epileptic medications. Remission usually occurs within 1-2 years from onset. One third have a single seizure but $5-10 \%$ may have more than 10 seizures or a more prolonged course. We present a 5 year old boy with this condition, who presented with episodes of severe vomiting, deviation of eyes, focal seizure, altered sensorium, characteristic EEG in the form of multiple occipital spikes and normal neuroimaging studies. [J Natl Inst Neurosci Bangladesh 2015;1(1): 27-30]
\end{abstract}

Keywords: Epilepsy; occipital; benign; pana yiotopoulos syndrome

Correspondence: Dr Md Enayet Hussain, Assistant Professor, Department of Neurology, National Institute of Neurosciences \& Hospital, Sher-E-Bangla Nagar, Agargaon, Dhaka, Bangladesh Email: enayetdmc@yahoo.com. Mobile: +8801716215871

Conflict of interest: None

Contribution to authors: RNC, MNI, AFMAMK \& MFM were involved in literature search and drafting the manuscript. MEH was involved in the management of the case and finalized the manuscript and will be the guarantor.

How to cite this article: Hussain ME, Chowdhury RN, Islam MN, Khan AFMAM, Miah MF. Early Onset Benign Occipital Epilepsy (Panayiotopoulos syndrome): a case Report. J Natl Inst Neurosci Bangladesh January 2015; 1(1): 27-30

\section{Introduction}

Panayiotopoulos syndrome is an idiopathic benign childhood focal epileptic syndrome recently recognized by the International League Against Epilepsy. It is characterized by seizures, often prolonged, with predominantly autonomic symptoms take nausea, vomiting, retching, pallor/cyanosis, pupillary changes, cardiorespiratory or thermoregulatory changes, incontinence of urine and less commonly feces and by an EEG that shows shifting and/or multiple foci, often with occipital predominance.

\section{Case report}

A 5 year old boy was brought to the Department of Neurology at NINSH for evaluation. The parents stated that the child was reasonably well when he had one episode of protracted vomiting while patient was sleeping at night. The child became so weak that the patient was unable to walk or even stand. The patient remained quiet in his bed. He was unresponsive to any call although his eyes were open and were turned to the left side. The patient had profuse salivation with drooling of saliva and was double incontinent. The patient was taken to a local hospital for resuscitation. The whole episode lasted for about one hour when the child gradually became almost normal with failure to recall the events in the preceding hour. The child again felt sick while he was playing. He started vomiting and became very pale. The parents noted jerky movement involving the left hand and twitching of the facial muscles on the left side which lasted for around 30 minutes. His eyes were turned towards the left side. He was again taken to the hospital where he was given inj phenobarbitone $(\mathrm{i} / \mathrm{m})$ which aborted the attack. The patient had 
incontinence of urine, vomited for 5-6 times. In the postictal period the patient was very weak, drowsy and tired. The whole episode lasted for about one hour. The child's intelligence is normal with normal mile stones of development. The patient is the second issue of a nonconsanguineous parents with no family history of seizure disorder. The patient was born by caesarean section with an uneventful antenatal, perinatal and post natal period. A clinical diagnosis of Panayiotopoulos syndrome was made and the patient was advised for an EEG (including sleep record) and MRI of the brain. The EEG revealed spikeand-wave complexes in the occipital (O1, O2) and posterior temporal (T5, T6) region which disappeared on eye opening (fixation off phenomenon) with a normal background.(Fig: 1). MRI was found to be normal. neurodevelopmental problems. Although it has been described as starting as early as 1 year of age and as late as 14 years of age, a notable feature is that most individuals have their first seizure around the age of 5 years. Threequarters of patients have their first seizure between the ages of 3 and 6 years. It affects males and females almost equally. ${ }^{2}$

Seizures in PS occur predominantly in sleep, particularly in the early part of sleep. This can be both nocturnal sleep and daytime naps. In two-thirds of patients, seizures occur only in sleep. The seizures of PS are characterized by autonomic symptoms. All functions of the autonomic nervous system can be affected, but emetic symptoms are the most obvious. Emetic symptoms may include one or more of

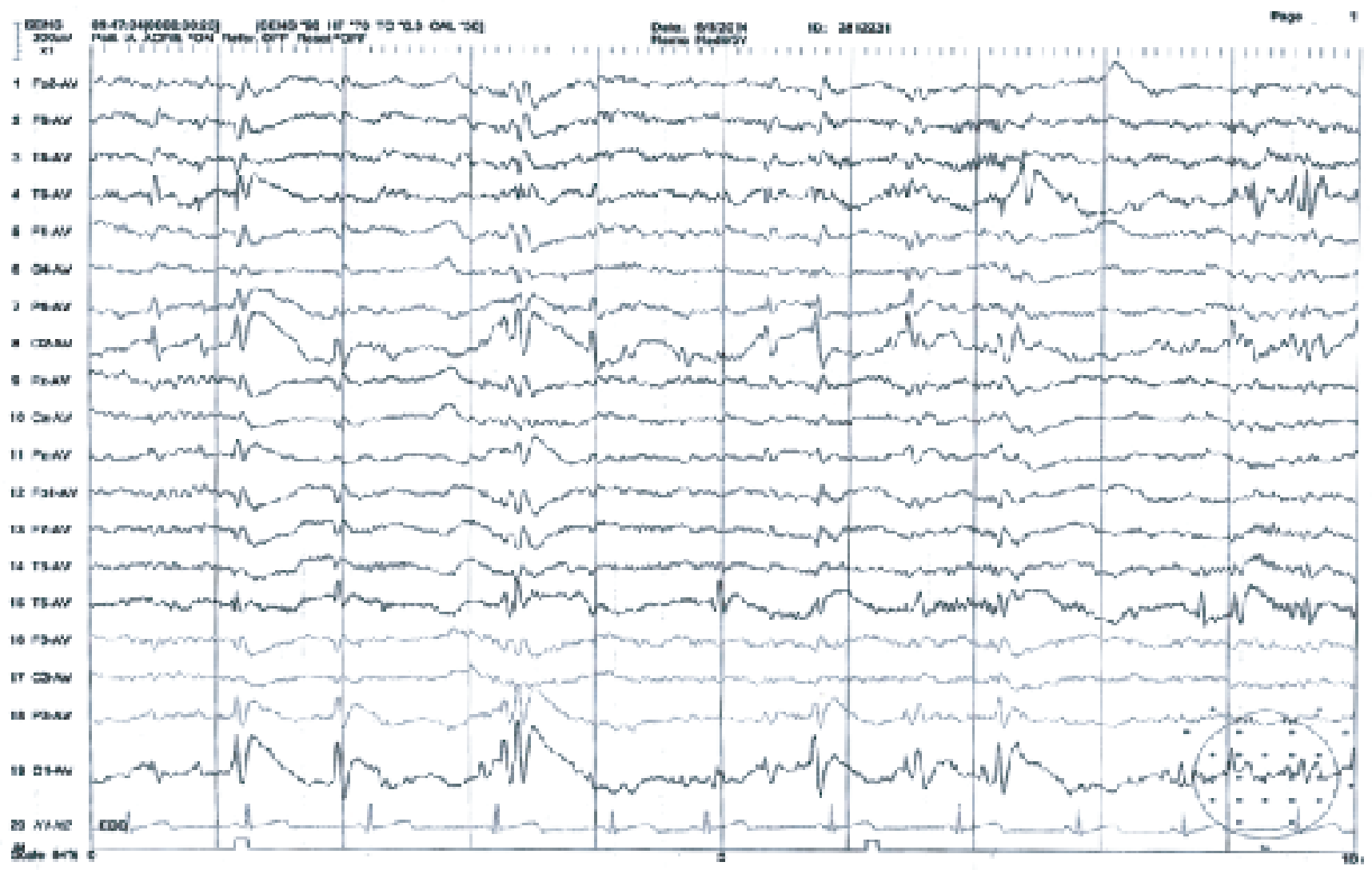

Figure 1: Posteriorly dominant Spike-and-wave in the occipital (O1,O2) \& posterior temporal (T5, T6) region

\section{Discussion}

Panayiotopoulos syndrome (PS) is an early onset benign focal epilepsy syndrome defined as a benign age-related autonomic epileptic disorder occurring in early and mid childhood with an EEG that shows shifting and/or multiple foci, often with occipital predominance. At least 5 of the criteria need to be present to make a diagnosis of Panayiotopoulos syndrome ${ }^{1}$ infrequent seizures ${ }^{2}$ prolonged seizures $>5$ minutes $^{3}$ ictal vomiting ${ }^{4}$ eye deviation ${ }^{5}$ autonomic manifestations ${ }^{6}$ behavioural disturbance and ${ }^{7}$ altered consciousness ${ }^{1}$.

PS occurs exclusively in children who are otherwise normal and is not associated with significant nausea, retching, and vomiting. In around three-quarters of patients, all three emetic symptoms are reported. Although vomiting is very common, it is not a prerequisite for diagnosis. When a seizure occurs during at awake. Statu initial stage the child is typically fully conscious, able to speak, but complains of feeling nauseated. If the seizure occurs in sleep, the child may awaken and complain of nausea, or may be found vomiting. In a minority cases of PS, features at idiopathic focal epilepsies of childhood may occur. Other autonomic features are also commonly present early in the seizure. These include colour changes, especially pallor, but sometimes flushing or cyanosis; pupillary changes, particularly mydriasis, less often miosis; coughing; cardiorespiratory and thermoregulatory 
alterations; and urinary, and less common faecal incontinence and modifications of intestinal mobility. Headache may be described and is generally concurrent with other autonomic symptoms. Details of the cardiorespiratory abnormalities, like apnoea and changes in heart rate, are sparse and require further study. In nearly all seizures, consciousness is initially intact but becomes impaired as the seizure progresses, with the child becoming confused or unresponsive. Deviation of the eyes and often the head to one side is common (around $60 \%$ of seizures), although it is not usually an early symptom, generally occurring after emetic symptoms. One-third of seizures terminate in hemi- or generalized convulsions. Some seizures in PS are manifested as the child becoming unresponsive and flaccid. This may occur without, or be followed by, convulsive features. Such attacks may suggest a syncope and the terms 'ictal syncope' or 'ictal syncopallike episodes', 'syncopal-like seizures', and 'fainting-like attacks' have been used to designate them. Usually, but not always, the syncopal- like features occupy a brief part of the seizure during which more usual autonomic and other features occur ${ }^{1,3}$.

Seizures in PS are typically long. Forty-four percent of seizures last 30 minutes or longer, constituting autonomic status epilepticus. Such events commonly terminate in hemi- or generalized convulsions. Convulsive status epilepticus is described but is exceptional. There are no recorded instances of residual neurological damage after seizures in PS.

PS is among the most benign of all seizure disorders. Around one-third of all patients will have a single seizure (hence the term 'seizure disorder' is preferred to epilepsy). Most will have between two and five seizures. A few have more than 10 seizures. Very frequent seizures are unusual but do not exclude the diagnosis. Seizures usually remit within 1-2 years of onset. Around one-fifth of patients develop other, usually infrequent, seizures during childhood and adolescence. These are usually Rolandic seizures and, less frequently, visual seizures; they all remit. The risk of epilepsy in adult life appears to be no higher than in the general population, although there is a need for more studies with long-term follow-up. Atypical evolution of PS with the development of absences and drop attacks has been described, but exceptiona ${ }^{12}$.

Panayiotopoulos syndrome may easily be missed or confused with many nonepileptic conditions. Mild ictal symptoms in the presence of clear consciousness would suggest trivial non-epileptic conditions such as atypical migraine, gastroenteritis or syncope, while prolonged and severe attacks may simulate life threatening insults such as encephalitis, for which many of these children are treated. Characteristically, even after the most severe symptoms the child becomes completely normal after a brief post ictal sleep. This is considered both diagnostic and reassuring ${ }^{5}$. The interictal EEG of PS shows a normal background with high-amplitude sharp and slow wave complexes. These are similar in morphology to those seen in benign childhood epilepsy with centrotemporal spikes. However, in PS there is great variability in their location. Occipital localization is the most common, but all other brain regions may be involved. Moreover, they frequently shift in location, this possibly being age-related. Brief generalized discharges are occasionally encountered. The sharp waves or sharp and slow wave complexes may repeat themselves more or less regularly and propagate, especially to frontal regions. EEG abnormalities in PS are accentuated by sleep $p^{4,8,9}$. Normal recordings may occur in $25 \%$ of children ${ }^{6}$.

The EEG in PS appears to indicate that the condition is associated with a diffuse/multifocal cortical hyperexcitability. This is also supported by magnetoencephalographic data $^{7}$. The predominance of interictal occipital EEG abnormalities suggests the possibility that the site of seizure onset is usually occipital. This is supported by the fact that three out of four reported ictal recordings had occipital onset. However, it seems likely that seizure onset may also be from extra-occipital sites. The clinical manifestations suggest that there is preferential spread to autonomic centres, including emetic centres or their cortical projections ${ }^{1}$.

Management: Education about the nature and prognosis of the syndrome is the cornerstone of correct management. Parents of children with the disorder should receive detailed information about the nature of the disorder and appropriate management of further seizures. Recommendations for antiepileptic drug treatment of PS come from case series. There are no randomized controlled studies. Prognosis, in terms of ultimate remission of seizures, does not appear to be affected by antiepileptic drug treatment. There is currently no evidence that the prolonged, non-convulsive seizures of PS pose a risk of damage. The seizures are often few in number. These features are similar to the situation with benign childhood epilepsy with centrotemporal spikes, in which it is now generally considered that antiepileptic drug treatment is not always required. Regular antiepileptic drug treatment in PS is probably most appropriately reserved for those children in whom seizures are unusually frequent or distressing or are otherwise significantly interfering in the life of the child. Prolonged seizures in PS can reasonably be treated with 'rescue' benzodiazepines (rectal diazepam or oral midazolam) as for febrile seizures ${ }^{2,4}$. There is no evidence that any particular antiepileptic drug is more efficacious than any other in PS. Of the established antiepileptic drugs, carbamazepine and sodium valproate appear to be equally appropriate. Given the benign nature of the syndrome, it is particularly important to avoid adverse effects; polypharmacy is rarely, if ever, indicated. The EEG should not be used as a guide to starting or stopping antiepileptic drug treatment. Given the short duration of active seizures in most children with PS, withdrawal after 1-2 seizure-free years is appropriate ${ }^{1}$. 


\section{Conclusion}

This case is an example of typical Panayiotopoulos syndrome. This is a relatively common benign focal childhood epilepsy. The recognition of the condition is important because in most cases reasurance and counselling about the benign nature of the disease to the parents is important. More over in most cases no antiepileptic medication is required.

\section{References}

1. Ferrie C, Caraballo R, Covanis A, Demirbilek V, Dervent A, Kivity S, et al Panayiotopoulos Syndrome: a consensus view. Dev Med Child Neurol 2006; 48:236-240.

2. Panayiotopoulos CP. Panayiotopoulos Syndrome: A common and Benign Childhood Epileptic Syndrome. London, United Kingdom: John Libbey \& Company, 2002

3. Panayiotopoulos CP. Vomiting as an ictal manifestation of epileptic seizures and syndromes. J Neurol Neurosurg Psychiat.1988;51:1448-145 4. Ferrie CD, Grunewald RA. Panayiotopoulos Syndrome: a common and benign childhood epilepsy. Lancet. 2001;357:821-823

5. Couvanis A. Panayiotopoulos: A benign autonomic epilepsy frequently imitating encephalitis, syncope, migraine, sleep disorder or gastroenteritis. AAP Grand Rounds 2006; 16: 69-70.

6. Koutroumanidis M, Ferrie CD, Sanders S, Rowlinson S. Panayiotopoulos syndrome of early onset benign childhood seizures: EEG variability and management issues. Clin Neurophysiol. 2001;112:1269.

7. Kanazawa O, Tohyama J, Akasaka N, Kamimura T. A magnetoencephalographic study of patients with Panayiotopoulos syndrome. Epilepsia 2005 46: 1106-1113.

8. Yalcin AD, Kaymaz A, Forta H. Childhood occipital epilepsy: seizure manifestations and electroencephalographic features. Brain Dev 1997 19: 408-413.

9. Yoshinaga H, Koutroumanidis M, Shirasawa A, Kikumoto K, Ohtsuka Y, Oka E. Dipole analysis in Panayiotopoulos syndrome. Brain Dev 2005 27: 46-52. 